\title{
Estudo da dependência energética de uma câmara de extrapolação em feixes de raios-x de baixa energia
}

\author{
F. M. Bastos ${ }^{\text {a }}$ T. A. da Silva ${ }^{\text {b }}$ \\ ${ }^{1}$ Curso de Pós-graduação em Ciência e Tecnologia das Radiações, Minerais e Materiais \\ Centro de Desenvolvimento da Tecnologia Nuclear, CDTN/CNEN \\ CEP 31270-90, Belo Horizonte-MG, Brasil \\ fernanda_mbastos@yahoo.com.br \\ ${ }^{2}$ Centro de Desenvolvimento da Tecnologia Nuclear, CDTN/CNEN \\ CEP 31270-901, Belo Horizonte-MG, Brasil \\ silvata@cdtn.br
}

\begin{abstract}
Este trabalho teve com principal objetivo estudar a dependência energética uma câmara de extrapolação em raios-X de baixa energia, a fim de determinar o valor da incerteza associada à variação da energia da radiação incidente nas medidas em que ela é utilizada Para o estudo de dependência energética, foram realizadas medidas de corrente de ionização comparativas entre a câmara de extrapolação e duas câmaras de ionização: uma câmara de mamografia, modelo RC6M, da Radcal, com dependência energética menor que 5\% e uma câmara de radioproteção modelo 2575 da NE Technology; ambas as câmaras possuem janelas muito finas, o que permite sua aplicação em feixes de baixas energias. As medidas foram feitas em quatro profundidades diferentes da câmara de extrapolação de 1,0 a 4,0 mm, em intervalo de 1,0 mm, para cada radiação de referência. O estudo evidenciou que há uma dependência energética variável com o volume da câmara de extrapolação. Em outra análise, conclui-se que a dependência energética da câmara de extrapolação torna-se menor quando se utiliza o coeficiente angular da reta de corrente de ionização versus profundidade, para as diferentes radiações de referência; isto demonstra que a técnica de extrapolação, utilizada para o cálculo de dose absorvida, diminui a incerteza associada à influência da variação da resposta com a energia da radiação.
\end{abstract}

\section{Keywords:}

Câmara de extrapolação, raios-X de baixa energia, dependência energética 


\section{INTRODUÇÃO}

$\mathrm{Na}$ área de dosimetria das radiações ionizantes, emprega-se o termo "radiações de referência" para designar características e métodos de produção de feixes de raios $\mathrm{X}$ e gama para calibração de dosímetros.

A International Standardization Organization (ISO) é uma organização internacional que estabelece quatro séries de radiações de referência para calibração e teste de desempenho de dosímetros: as séries L (Low) e $\mathrm{H}$ (High) respectivamente em baixa e alta taxa de kerma no ar, as séries N (Narrow) de espectro estreito e a série W (Wide) de espectro largo. A caracterização dos feixes de raios-X de referência é feita pela determinação dos parâmetros: camada semirredutora, energia média, resolução espectral, tensão do tubo e filtrações [1].

Câmaras de extrapolação são câmaras de ionização que possuem uma característica que as torna única para realização de medidas de dose absorvida: um dos eletrodos paralelos pode ser movimentado, permitindo que a massa do volume sensível no interior do detector seja variável e com isso extrapolar o valor da corrente de ionização para um volume infinitamente pequeno e estimar o valor da dose absorvida em tecido de espessura fina como, por exemplo, a pele [2]. Tanto câmaras de extrapolação projetadas e construídas [3] quanto as disponíveis comercialmente [4] precisam ter suas características metrológicas determinadas antes da sua utilização. Este trabalho objetivou estudar a variação da resposta da câmara de extrapolação PTW modelo 23392 para raios-X de referência inferiores a $30 \mathrm{keV}$. A dependência energética da câmara foi analisada tanto para volumes fixos quanto utilizando a técnica de extrapolação para volume nulo. 


\section{MATERIAIS E MÉTODOS}

Este trabalho foi desenvolvido no Laboratório de Calibração de Dosímetros (LCD), localizado no Centro de Desenvolvimento da Tecnologia Nuclear (CDTN/CNEN), em Belo Horizonte. O estudo foi feito na câmara de extrapolação PTW-Freiburg, modelo 23392, que possui janela de entrada em filme de poliéster Mylar®, revestida de grafite com diâmetro de 60,5 mm; eletrodo coletor de PMMA com superfície revestida por grafite com diâmetro de $30 \mathrm{~mm}$ e distância entre os eletrodos variável de 0,5 a $10,5 \mathrm{~mm}$, medida a partir de um micrômetro com precisão de \pm 1 $\mu \mathrm{m}$, caracterizando um volume variável de 0,353 a $7,422 \mathrm{~cm}^{3}$.

As radiações de referência das séries $\mathrm{L}, \mathrm{H}$ e N, com energias médias inferiores a $30 \mathrm{keV}$, foram reproduzidas na máquina de raios-X industrial Pantak-Seifert, modelo ISOVOLT HS320 de potencial constante, cujo tubo possui uma janela de $7 \mathrm{~mm}$ de berílio. As características dos feixes implantados no LCD/CDTN ${ }^{[5]}$ são apresentadas na Tabela 1.

Tabela 1: Características das radiações $X$ de referência de baixa energia da norma ISO4037 implantadas no LCD/CDTN.

\begin{tabular}{cccccccc}
\hline Qualidade & $\begin{array}{c}\text { Tensão } \\
(\mathrm{kV})\end{array}$ & $\begin{array}{c}\text { Energia } \\
\text { média } \\
(\mathrm{keV})\end{array}$ & \multicolumn{2}{c}{ Filtração Adicional } & $\begin{array}{c}1^{\text {a CSR }} \\
(\mathrm{mm} \mathrm{Al})\end{array}$ & $\begin{array}{c}2^{\text {a }} \text { CSR } \\
(\mathrm{mm} \mathrm{Al})\end{array}$ \\
\hline $\mathbf{L 1 0}$ & 10,2 & 8,5 & 0,04 & - & 1000 & 0,059 & - \\
$\mathbf{L 2 0}$ & 20,2 & 17,3 & 2,00 & - & 1000 & 0,416 & - \\
$\mathbf{L 3 0}$ & 30,0 & 26,4 & 3,60 & 0,178 & 1000 & 1,450 & - \\
$\mathbf{N 1 0}$ & 10 & 8,2 & 0,04 & - & 1000 & 0,049 & 0,051 \\
$\mathbf{N 1 5}$ & 15 & 12,0 & 0,52 & - & 1000 & 0,145 & 0,162 \\
$\mathbf{N 2 0}$ & 20 & 15,8 & 0,85 & - & 1000 & 0,320 & 0,360 \\
$\mathbf{N 2 5}$ & 25 & 19,9 & 2,00 & - & 1000 & 0,650 & 0,750 \\
$\mathbf{N 3 0}$ & 30 & 23,9 & 4,00 & - & 1000 & 1,150 & 1,300 \\
\hline
\end{tabular}




\begin{tabular}{llllllll}
\hline H10 & 10 & 8,8 & - & - & 500 & 0,039 & - \\
H20 & 20 & 14,4 & 0,06 & - & 1000 & 1,140 & - \\
H30 & 30 & 20,3 & 0,42 & - & 1000 & 0,357 & - \\
\hline
\end{tabular}

O estudo da dependência energética da câmara de extrapolação PTW 23392 foi feito de forma comparativa com uma câmara Radcal, modelo RC-6M, de 6 cm³ e uma câmara NE Technology, modelo 2575, de $600 \mathrm{~cm}^{3}$, ambas com janelas de entrada muito finas, o que permite sua aplicação em feixes de baixas energias.. O campo elétrico aplicado entre os eletrodos da câmara de extrapolação PTW 23392 foi mantido constante em 70 V/mm por meio de um eletrômetro da Keithley; as tensões aplicadas na Radcal RC-6M e na NE 2575 foram $300 \mathrm{~V}$ e $-250 \mathrm{~V}$, respectivamente, produzidas pelo eletrômetro PTW-Unidos.

Os três detectores foram posicionados de forma que, pelo método de substituição, cada janela estivesse a $100 \mathrm{~cm}$ do tubo de raios-X (a $50 \mathrm{~cm}$ para a qualidade H10), no centro do mesmo campo de radiação. $\mathrm{O}$ arranjo experimental para medidas comparativas entre as câmaras é mostrado na Figura 1.

Figura 1. Arranjo experimental para comparação da dependência energética em feixes de raios-X de baixa energia da câmara de extrapolação PTW 23392 (centro) com as câmaras Radcal RC-6M (direita) e NE 2575 (esquerda).

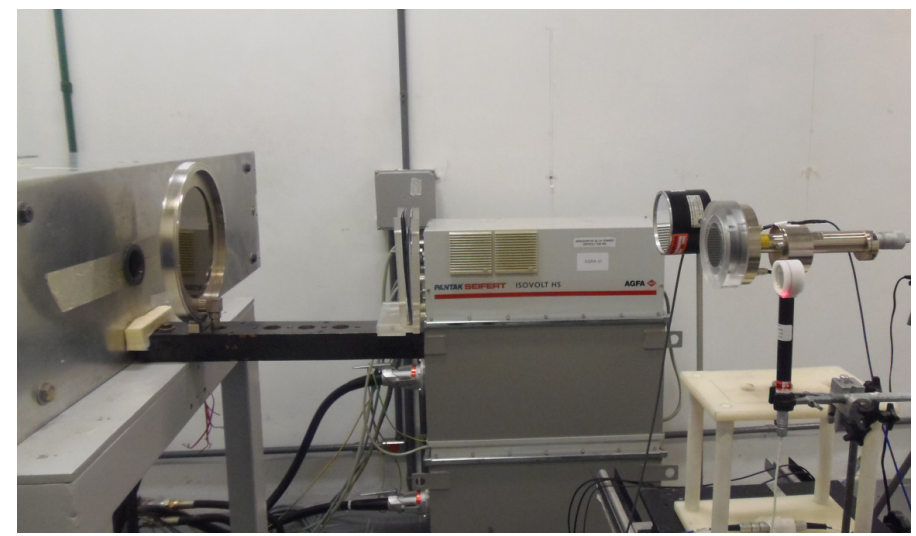




\section{RESULTADOS E DISCUSSÕES}

As três câmaras de ionização foram comparadas em todas as qualidades de feixe da Tabela 1, mantendo-se a taxa de Kerma no ar constante. Para cada condição, foram realizadas cinco leituras da corrente de ionização e os valores corrigidos para condições ambientais de referência de $20{ }^{\circ} \mathrm{C}$ e $101,325 \mathrm{kPa}$.

A dependência energética da câmara de extrapolação PTW 23392 foi analisada de forma relativa, a partir da razão entre a corrente de ionização desta com a da câmara Radcal RC-6M e com a da câmara NE 2575. Além disso, para o estudo de dependência energética de uma câmara de ionização, é necessário normalizar as razões de correntes de ionização. Para isso, dividem-se as razões de correntes de todas as radiações de uma qualidade pelo valor de razão de correntes de uma radiação de referência determinada. As qualidades $\mathrm{N}$ foram normalizadas para a radiação de referência N25, as qualidades L para L20 e as qualidades H para H30.

\subsection{Dependência energética da câmara PTW 23392 com volumes fixos}

A Figura 2 apresenta os resultados normalizados comparativos da câmara PTW 23392, com distâncias entre os eletrodos de 1,0 a 4,0 mm, com a câmara Radcal RC-6M, nas qualidades N, L e H. É possível observar que, para todas as qualidades, a dependência energética da câmara PTW 23392, quando comparada a da câmara Radcal RC-6M, tende a diminuir com o aumento da distância entre os eletrodos de 1,0 para 4,0 mm. Na qualidade N, a redução foi de $15 \%$ para $8 \%$ relativa à radiação N25 (energia de 19,9 keV); na qualidade L, a redução foi de $16 \%$ para 8\%, relativa à radiação L20 (energia de 17,3 keV) e na qualidade $\mathrm{H}$, de $13 \%$ para menos de $2 \%$, relativa à radiação $\mathrm{H} 30$ (energia de $20,3 \mathrm{keV}$ ). 

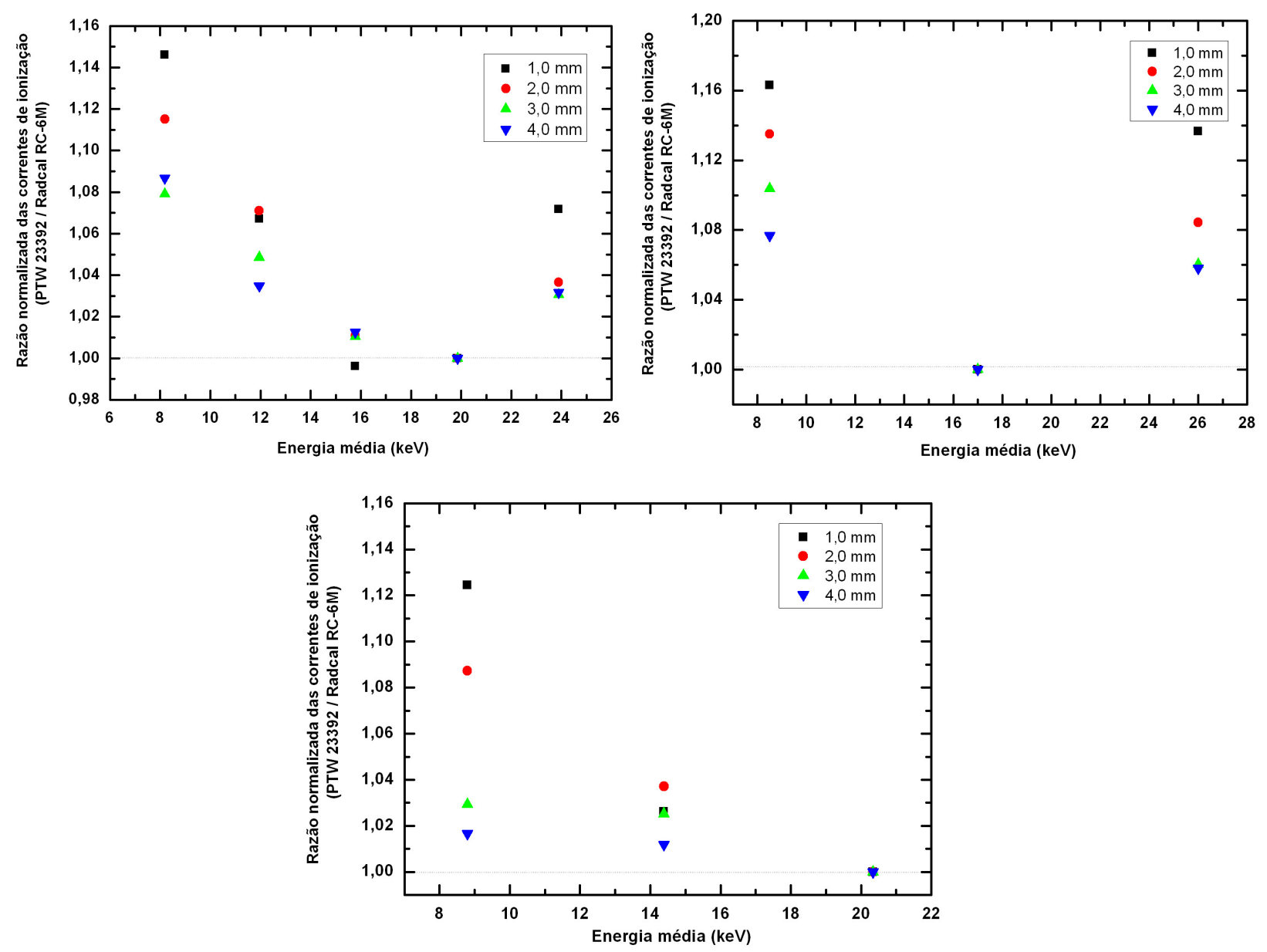

Figura 2. Dependência energética da câmara de extrapolação PTW 23392, com diferentes distâncias entre os eletrodos, comparada à câmara Radcal RC-6M, para as radiações de referência $N$ (acima à esquerda, normalizada para N25), L (acima à direita, normalizada para L20) e H (abaixo, normalizada para H30).

A Figura 3 apresenta, de forma similar, os resultados comparativos entre a câmara PTW 23392 e a NE2575. Como esperado, os resultados apresentaram o mesmo comportamento, evidenciando a redução da dependência energética da câmara de extrapolação PTW 23392 à medida que a distância entre os eletrodos aumentou de 1,0 para 4,0 $\mathrm{mm}$. Na qualidade $\mathrm{N}$ a redução foi de $22 \%$ para $14 \%$ relativa à radiação N25 (energia de $19,9 \mathrm{keV}$ ); na qualidade $\mathrm{L}$, a redução foi de $21 \%$ 
para $12 \%$, relativa à radiação L 20 (energia de $17,3 \mathrm{keV}$ ) e na qualidade $\mathrm{H}$, de $32 \%$ para $18 \%$, relativa à radiação $\mathrm{H} 30$ (energia de $20,3 \mathrm{keV}$ ).
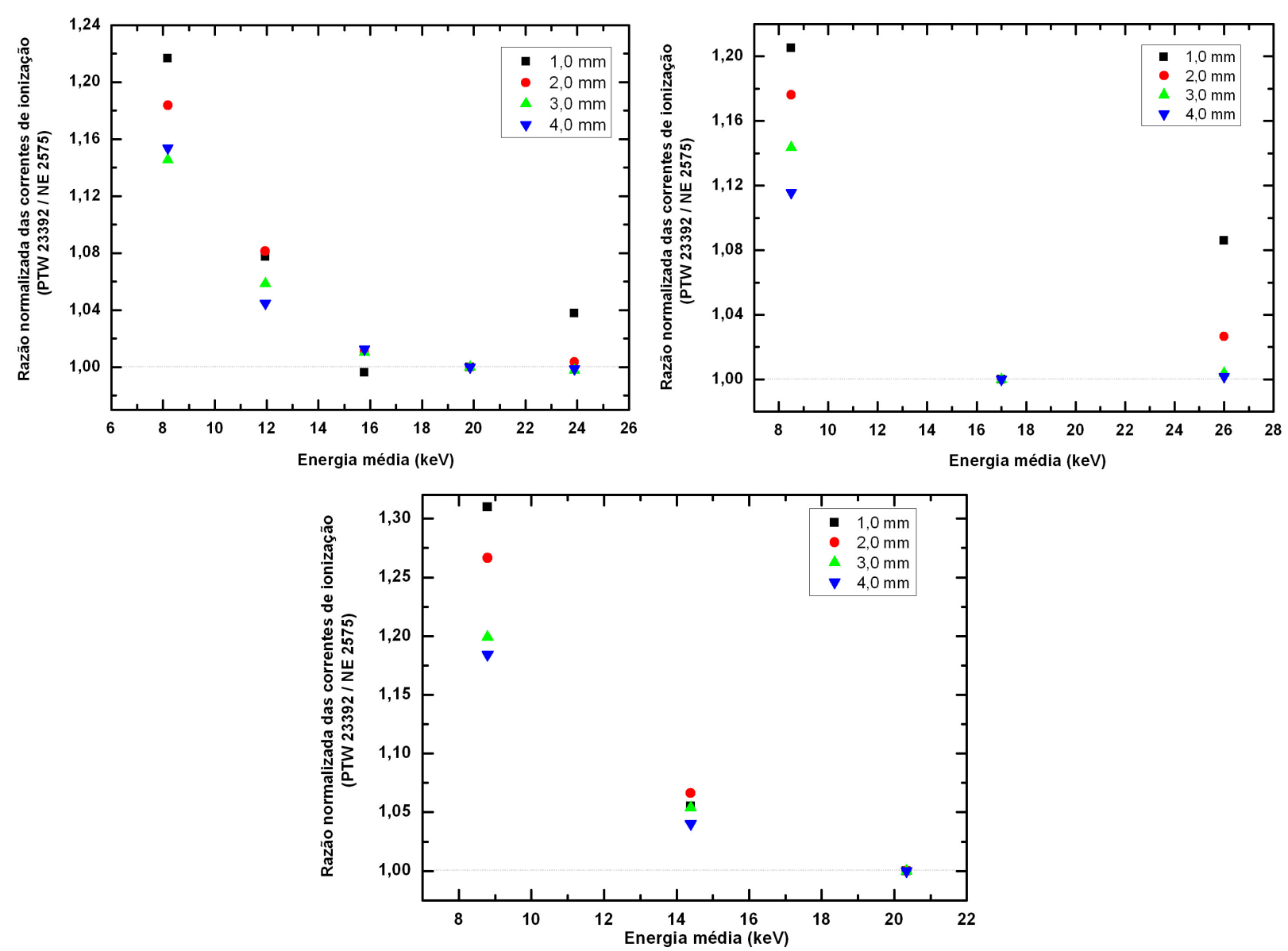

Figura 3. Dependência energética da câmara de extrapolação PTW 23392, com diferentes distâncias entre os eletrodos, comparada à câmara NE 2575, para as radiações de referência $N$ (acima à esquerda, normalizada para N25), L (acima à direita, normalizada para L20) e H (abaixo, normalizada para H30).

A maior dependência energética da câmara PTW 23393 em relação à NE 2575 (Fig. 3) em comparação à Radcal RC-6M (Fig. 2) evidencia a diferença de dependência energética entre as 
duas câmaras usadas para comparação; possivelmente a NE 2575 possui maior dependência energética do que a Radcal RC-6M.

Os resultados (Fig. 2 e 3) sugerem que, para reduzir a influência da dependência energética da câmara de extrapolação PTW 23393, em medidas de camada semirredutora, por exemplo, devese usar a câmara com 4,0 mm de distância entre os eletrodos.

\subsection{Dependência energética da câmara PTW 23392, com a técnica de extrapolação}

Usando-se a técnica de extrapolação, foi determinado o coeficiente angular da reta de extrapolação para cada energia de feixe de raios-X de referência. A Figura 4 apresenta os resultados comparativos da câmara PTW 23392 com a câmara Radcal RC-6M e com a NE 2575, nas qualidades $\mathrm{N}$ (o coeficiente foi divido pela corrente de ionização de cada câmara de referência). Os resultados mostraram que a dependência energética da câmara PTW 23392 foi de $6,5 \%$ e de $13 \%$, relativa à câmara Radcal RC-6M e à NE 2575, respectivamente normalizada para a radiação de referência N25 (energia de 19,9 keV). A dependência energética da câmara PTW 23392, usando a técnica de extrapolação, foi menor que aquela determinada para volumes fixos com distâncias entre os eletrodos de 1,0 a 3,0 mm.

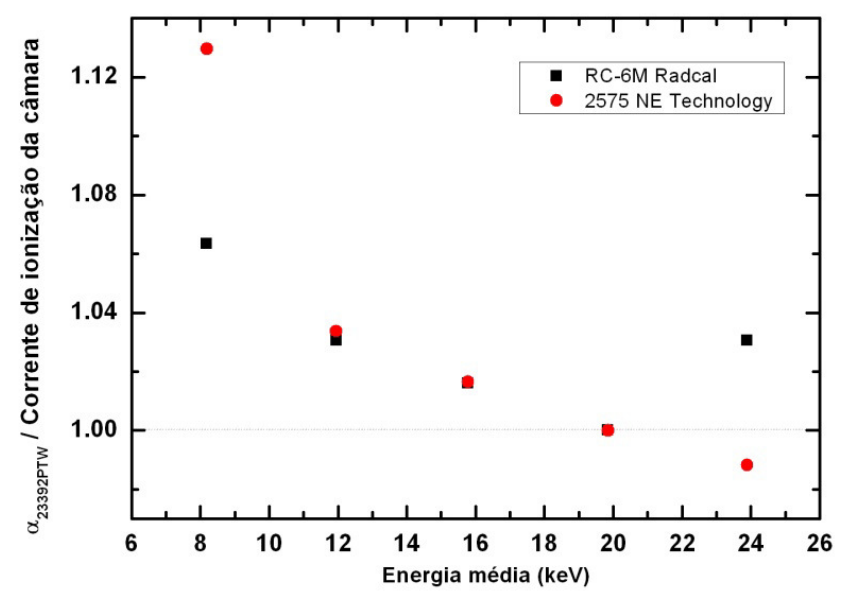

Figura 4. Dependência energética da câmara de extrapolação PTW 23392, com base no coeficiente angular $\left(\alpha_{P T W 23392}\right)$, comparada à câmara Radcal RC-6M e à câmara NE 2575, para as radiações de referência $N$ (normalizada para $N 25$ ). 


\section{CONCLUSÕES}

A dependência energética da câmara de extrapolação PTW 23392 foi determinada comparativamente as duas câmaras, apresentando valores inferiores a 10\%, se adotada a técnica de extrapolação. A adoção da técnica reduzirá, também, outros efeitos indesejáveis como espalhamento da radiação e falta de repetibilidade nas medidas de baixos valores de corrente de ionização.

Esta caracterização da câmara de extrapolação é relevante, para seu uso em medidas da camada semirredutora de feixes de baixa energia.

\section{AGRADECIMENTOS}

Fernanda Martins Bastos agradece à CNEN pela bolsa de mestrado. Este trabalho foi apoiado pela FAPEMIG (PPM) e CNPQ (PQ) e faz parte do projeto INCT Metrologia das Radiações em Medicina. 


\section{REFERÊNCIAS}

1. ISO - International Standerdization Organization. $\mathbf{X}$ and gamma reference radiation for calibrating dosemeters and dose rate meters and for determining their response as a function of photon energy. ISO 4037. Geneva, Suiça: ISO, 1996.

2. BOHM, J. e U. SCHNEIDER. Review of extrapolation chamber measurements of beta rays and low energy rays. Radiation Protection Dosimetry. v. 14 (2), pp. 193-198, 1986.

3. DA SILVA, E. A. B. e CALDAS, L. V. E. Application of extrapolation chambers in lowenergy X-rays as reference systems. Applied Radiation and Isotopes. v 70, pp. 1411-1416, 2012.

4. PTW-FREIBURG. 2002. Instruction Manual Extrapolation Chamber according to Böhm Type 23392. Freiburg, Germany, 2002.

5. TADEU, M. “Análise dos Procedimentos e Critérios de Implatação de Feixes de Raios-X de Refêrencia ISO 4037 em baixas energias” Dissertação de Mestrado. Belo Horizonte: Centro de Desenvolvimento da Tecnologia Nuclear/CNEN, 2012. 